\title{
Brief behavioural surveys in routine HIV sentinel surveillance: a new tool for monitoring the HIV epidemic in Viet Nam
}

\author{
Duong Cong Thanh, ${ }^{a}$ Nguyen Tran Hien, ${ }^{a}$ Nguyen Anh Tuan, ${ }^{a}$ Hoang Thi Thanh Ha, ${ }^{a}$ Pham Hong Thang, \\ Nguyen Thi Thanh Ha, ${ }^{a}$ Le Anh Tuan, ${ }^{a}$ Tran Dai Quang, ${ }^{a}$ Tran Hong Tram, ${ }^{a}$ Nguyen Le Hai, ${ }^{a}$ \\ Phan Thi Thu Huong, ${ }^{b}$ Vo Hai Son, ${ }^{b}$ Bui Hoang Duc, ${ }^{b}$ Nguyen Thien Nga, ${ }^{c}$ David Jacka ${ }^{c}$ and Keith Sabin ${ }^{c}$ \\ Correspondence to Duong Cong Thanh (e-mail: congthanhnihe@yahoo.com).
}

In this report we describe a new approach in HIV sentinel surveillance that was piloted in Viet Nam in 2009 and is currently being rolled out in all provinces. It comprises a brief behavioural questionnaire added to the HIV sentinel surveillance surveys conducted routinely among people who inject drugs, female sex workers and men who have sex with men. Timely reporting of data from this system has resulted in improvements to HIV prevention efforts for most at-risk populations.

\section{Brief History of HIV Surveillance in Viet Nam}

HIV testing started in Viet Nam in 1988 in four large cities: Ha Noi, Hai Phong, Da Nang and Ho Chi Minh City and included all provinces in 1996. The HIV sentinel surveillance (HSS) system commenced in 10 provinces in 1994 to monitor the HIV seroprevalence annually among people who inject drugs (PWID), female sex workers (FSWs), men who have sex with men (MSM), sexually transmitted infection (STI) patients, antenatal women and army recruits. ${ }^{1}$ Over time HSS was expanded to more provinces and in 2014 was completed in 40 provinces.

In 2000 and 2001, behavioural surveillance surveys were conducted to monitor behaviour of PWID and FSWs in five large provinces, ${ }^{2}$ and Integrated Behavioural and Biological Surveys (IBBS) were conducted in 2005-2006, 2009 and 2013 to measure the prevalence of HIV, other STIs and key HIV risk and preventive behaviour among PWID, FSWs and MSM. ${ }^{3-5}$ Although IBBS provided rich and rigorous data, it is costly and time-consuming to conduct the data collection, analysis, report preparation and dissemination of results.

\section{New Approach in HIV Sentinel Surveillance}

For more timely data that can be readily used, Nha Trang Pasteur Institute in collaboration with the World Health Organization piloted the feasibility of a brief behavioural questionnaire added to HSS among PWID, FSWs and MSM in 2009. Since 2010, the National Institute of Hygiene and Epidemiology has continued this pilot to determine its feasibility and practicality, to monitor trends of key indicators of risk behaviour and to assess coverage of essential HIV/AIDS services. The system became known as HSS $+{ }^{6}$ The positive experiences of the pilot provinces led to an expansion to 12 provinces in 2011, 29 provinces in 2012 and 23 provinces in 2013.

Each HSS + survey comprises sample sizes from 150 to 300 , with multistage cluster sampling applied to recruit participants. Clusters were identified through residential communes for PWID and hotspots for FSWs and MSM. Eligible participants were asked to consent to a brief questionnaire and a blood draw and were offered a referral to the local HIV/AIDS services. The questionnaire was simple, covering all global and national

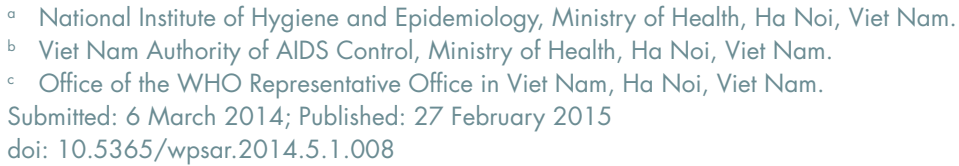


indicators (Table 1). A Standard Operating Procedures was developed, ${ }^{7}$ and provincial AIDS centres' staff were trained on data collection and reporting. An Epi-Data file and report template were provided to each provincial AIDS centre for data entry and report writing.

\section{Key Findings of HSS+}

As a result of the new HSS+, individual provincial reports were submitted within one month, and the national report was completed within two months of data collection. Additional funding (US\$ 8000 per province, which is $10 \%$ of IBBS funding) was provided for the additional fieldwork time needed by HSS staff. Acceptance by the participating populations has been high according to the interviewers. Despite this, the routine field monitoring and technical assistance revealed that there was a lack of adherence to national protocols by local staff in sampling, participant screening and interviewing; following time frame and skip patterns may affect the quality of the data. Supervision of staff is variable and provincial staff capacities differ.

In 2010, 2011 and 2012, respectively, 7\%, 3\% and $2 \%$ of FSWs reported injecting drugs. Of these, $82 \%, 81 \%$ and $70 \%$ reported having injected drugs in the past month. HIV prevalence among drug-injecting FSWs was $29 \%, 22 \%$ and $16 \%$ compared with the HIV prevalence among all FSWs at $4 \%, 3 \%$ and $3 \%$. This disproportionate risk of infection among FSWs who inject drugs resulted in the expansion of needle and syringe access for FSWs through peer outreach programmes. $^{8}$
From HSS+ data it also was found that among 1399 MSM participants in An Giang, Da Nang, Ha Noi, Hai Duong and Ho Chi Minh City in 2011, 3\% of MSM respondents reported injecting drugs in the last month, and $19 \%$ reported selling sex in the past 12 months. These groups had an HIV prevalence of $7 \%$ and $27 \%$, respectively. This diversity of risk among the MSM population led to new approaches and a refocusing of prevention efforts for MSM: strengthening HIV voluntary counselling and testing with focus on MSM who sell sex; needle and syringe programmes; and methadone mainternance treatment for drug-injecting MSM. ${ }^{8}$

\section{Conclusions}

HSS + offers a reasonably cost effective approach to obtaining data on HIV risk behaviour within the standard HSS system. The richness and timeliness of the data for monitoring and evaluating provincial programmes is generating high-level interest. A comparison between HSS + and IBBS will not be able to establish one survey as more valid than the other; however, the timeliness and utility of HSS + data have crystallized support by a donor community anxious for timely data.

\section{Next Steps}

Viet Nam is revising the HSS+ protocol to enhance sampling and improve supervision. As provincial staff come to a better understanding of how the data can help their efforts in prevention and control of HIV in their locality, their receptiveness to protocols should increase. With the HSS + approach in Viet Nam, we can monitor

Table 1. UNGASS and national indicators for HIV/AIDS monitoring and evaluation programme

\begin{tabular}{|c|c|c|}
\hline Indicators & UNGASS indicator $^{9}$ & National indicator \\
\hline FSWs using condoms with the most recent client & $x$ & $x$ \\
\hline FSWs using condoms regularly/consistently with clients in the past month & & $x$ \\
\hline FSWs injecting drug in the past month & & $x$ \\
\hline PWID sharing needles and syringes in the past month & $\mathrm{x}$ & $\mathrm{x}$ \\
\hline PWID using condoms during the most recent sexual intercourse & $\mathrm{x}$ & $x$ \\
\hline MSM using condoms during the most recent sexual intercourse with male partner & $x$ & $x$ \\
\hline PWID using clean needles and syringes in the most recent injection & $x$ & $x$ \\
\hline $\begin{array}{l}\text { FSWs, PWID and MSM being approached by the harm reduction prevention } \\
\text { programmes in the last six months }\end{array}$ & $x$ & $x$ \\
\hline FSWs, PWID and MSM being examined and treated for STIS & & $\mathrm{x}$ \\
\hline FSWs, PWID and MSM being tested and known the results in the past 12 months & $x$ & $x$ \\
\hline
\end{tabular}

FSW, female sex worker; MSM, men who have sex with men; PWID, people who inject drugs; STI, sexually transmitted infection; and UNGASS, United Nations General Assembly Special Session on HIV/AIDS. 
progress in more provinces on an annual basis for a relatively low cost.

\section{Conflicts of interest}

None declared.

\section{Funding}

None.

\section{References:}

1. Decision No. 1418: Guideline on HIV/STI surveillance in Viet Nam [In Vietnamese]. Ha Noi, Ministry of Health, 2000 (http://www.vaac.gov.vn:80/Desktop.aspx/Van-ban-phap-quy/ Bo-Y-te/C6D150DEDBA5467CA2F0DC5F05AEC092/, accessed 10 February 2015).

2. Results from the Behavioral Surveillance Survey (BBS) in Viet Nam. Ha Noi, Viet Nam Administration for AIDS Control, Ministry of Health, 2002.

3. Results from the HIV/STI Integrated Biological and Behavioral Surveillance in Viet Nam. Ha Noi, Viet Nam Administration for AIDS Control, Ministry of Health, 2007 (http://www.aidsdatahub.org/sites/default/files/documents/ Results_from_the_from_the_IBBS_in_Vietnam_2006.pdf.pdf, accessed 10 February 2015).

4. National Institute of Hygiene and Epidemiology. Results from the HIV/STI Integrated Biological and Behavioral Surveillance in
Viet Nam - Round II. Ha Noi, Ministry of Health, 2011 (http:// www.nihe.org.vn/new-en/chuong-trinh-phong-chong-sot-xuathuyet-8592614297/2744/Results-from-the-HIVSTI-integratedbiological-and-behavioral-surveillance-IBBS-in-Vietnam--RoundII--2009.vhtm, accessed 10 February 2015).

5. National Institute of Hygiene and Epidemiology. Results from the HIV/STI Integrated Biological and Behavioural Surveillance in Viet Nam - Round IIsI (Draft). Ha Noi, Ministry of Health, 2014.

6. Circular No 09: Guideline on HIV/STI surveillance in Viet Nam [In Vietnamese]. Ha Noi, Vietnam Administration for AIDS Control, Ministry of Health, 2012 (http://www. vaac.gov.vn:80/Desktop.aspx/Van-ban-phap-quy/Bo-Yte/B49D8A7D38BB47938E82CE609959BC04/, accessed 10 February 2015).

7. National Institute of Hygiene and Epidemiology. Standard operating procedures for HIV/STI sentinel surveillance plus behavioral component in Vietnam [In Vietnamese]. Ha Noi, National Institute of Hygiene and Epidemiology, 2014 (http://www. nihe.org.vn/uploads/SOP-HSS-NIHE-9June14-final.pdf, accessed 10 February 2015).

8. National strategy for HIV/AIDS prevention and control to 2020 and vision to 2030 [In Vietnamese]. Ha Noi, Government Cabinet, 2012 (http://www.nihe.org.vn/new-vn/chuong-trinh-giam-sat-theodoi-va-danh-gia-nhiem-h/2876/Chien-luoc-quoc-gia-Phongchong-HIVAIDS-den-nam-2020-va-tam-nhin-2030.vhtm, accessed 10 February 2015).

9. New UNGASS Guidelines for 2010 reporting. Geneva, UNAIDS, 2009 (http://data.unaids.org/pub/Manual/2009/JC1676_Core Indicators_2009_en.pdf, accessed 10 February 2015). 